\section{Decontamination of knives used in a slaughterhouse by a commercial non-thermal UV-C treatment}

\author{
Susanne Raschle, Claudio Zweifel, \\ Katrin Zurfluh, Roger Stephan
}

Institute for Food Safety and Hygiene, Vetsuisse Faculty, University of Zurich, Switzerland

\begin{abstract}
To assess the antimicrobial effect of a commercial UV-C system, knives inoculated with Escherichia coli and Staphylococcus aureus as well as naturally contaminated and collected from the wet and clean area of a slaughterhouse knives were examined. For inoculated knives, UV$\mathrm{C}$ treatment for $30 \mathrm{~s}$ reduced mean $E$. coli counts by $5.1 \log \mathrm{CFU} \mathrm{cm} \mathrm{cm}^{-2}$ and mean $S$.

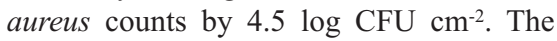
presence of blood lowered mean reductions to $3.4 \log \mathrm{CFU} \mathrm{cm}{ }^{-2}$ for $E$. coli and to 2.5 $\log \mathrm{CFU} \mathrm{\textrm {cm } ^ { - 2 }}$ for $S$. aureus. The presence of fat had a greater negative impact on the efficacy of the UV-C treatment resulting in

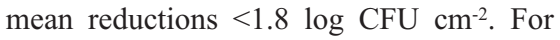
naturally contaminated knives from a slaughterhouse, total viable counts (TVC) before UV-C treatment varied considerably

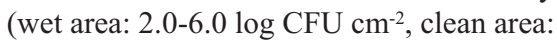
1.0-3.0 $\log \mathrm{CFU} \mathrm{\textrm {cm } ^ { - 2 }}$ ). UV-C treatment for 30 s reduced mean TVC by $0.8 \log \mathrm{CFU} \mathrm{\textrm {cm } ^ { - 2 }}$

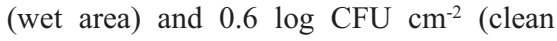
area), but the effect varied greatly between individual knives. Thus, under commercial conditions, the antibacterial effect of UV-C for the decontamination of knives is affected by the presence of additional contaminations like blood or fat. The adequate cleaning of the knives prior to UV-C decontamination is therefore of central importance.
\end{abstract}

\section{Introduction}

Strict adherence to good practices of slaughter hygiene along with risk-based preventive measures (HACCP approach) is crucial to ensure food safety at slaughter (Nørrung \& Buncic, 2008; Sofos, 2008). Because healthy food-producing animals can be carriers of bacterial pathogens causing human disease (EFSA/ECDC, 2017). Despite all advancements in slaughter technologies, complete prevention of microbial contamination of carcasses during slaughter can hardly be warranted. To limit contamination by equipment and utensils like knives, an effective cleaning and disinfection regime is of great importance.

For knives used during slaughter, inprocess cleaning and disinfection should be performed after each use. The most widespread procedure also mentioned in the European legislation (EU Regulation $853 / 2004)$ is hot-water decontamination at $82^{\circ} \mathrm{C}$ in a water bath. Its efficacy is affected by various framing conditions and limitations (Eustace et al., 2008; Goulter et al., 2008; Leps et al., 2013; Taormina \& Dorsa, 2007), which include but are not limited to: The presence of organic matter (which has to be removed prior cleaning); the hot-water exposure time (on which there is no consensus); the maintenance of the required hotwater temperature (leading to high energy consumption and steam formation); the affected sharpness and increased wear of the knives. Hence, there is interest in (nonthermal) alternatives for knife decontamination and the legal framework allows the use of alternatives with an equivalent effect to hot-water treatment (EU Regulation $853 / 2004)$. Various alternatives to hot-water treatment and combinations of hot water with chemicals have been evaluated for knife decontamination in the meat industry (Barbosa et al., 2016; Brasil et al., 2017; Eustace et al., 2008; Leps et al., 2013; Taormina \& Dorsa, 2007). Ultraviolet (UV) light is commonly used in the food industry for the decontamination of surfaces (e.g. packaging material), water, or air (Bintsis et al., 2000) and it could constitute a promising, non-thermal alternative for knife decontamination. The UV spectrum can be subdivided into a number of ranges: The long-wave UV-A (315-400 nm, "blacklight"), the medium-wave UV-B (280-315 $\mathrm{nm}$, responsible for human sunburn), and the short-wave UV-C (200-280 nm). The UV-C rays have an antimicrobial (germicidal) effect at a wavelength of $254 \mathrm{~nm}$. However, studies investigating the effect of UV-C for the decontamination of knives used in the meat industry are lacking in the literature, in particular studies performed under routine operations during slaughter. Therefore, the aim of the present study was therefore to determine the bacterial reductions obtained by a commercial non-thermal UV-C system (i) on knives inoculated with Escherichia (E.) coli or Staphylococcus (S.) aureus (with and without additional contamination) and (ii) on naturally contaminated knives under commercial conditions in a slaughterhouse.
Correspondence: Roger Stephan, Institute for Food Safety and Hygiene, Vetsuisse Faculty University of Zurich, Winterthurerstrasse 272, 8057 Zurich, Switzerland.

Tel.: +41.44.6358651 - Fax: +41.44.6358908 E-mail: roger.stephan@uzh.ch

Key words: UV-C decontamination; Knives; Inoculation; Natural contamination; Slaughterhouse.

Acknowledgments: The authors thank the staff of the slaughterhouse involved in this study for facilitating access to their operations and for the assistance with the collection of data.

Contributions: the authors contributed equally.

Conflict of interest: the authors declare no potential conflict of interest.

Funding: none.

Received for publication: 12 February 2019.

Revision received: 22 April 2019.

Accepted for publication: 29 April 2019.

This work is licensed under a Creative Commons Attribution-NonCommercial 4.0 International License (CC BY-NC 4.0).

CC Copyright S. Raschle et al., 2019

Licensee PAGEPress, Italy

Italian Journal of Food Safety 2019; 8:8107

doi:10.4081/ijfs.2019.8107

\section{Materials and Methods}

\section{UV-C decontamination device}

For UV-C decontamination of knives, the ME3-S device (Sterilsystems, Mautendorf, Austria) with dimensions of $215 \mathrm{~mm}$ (width) $\times 600 \mathrm{~mm}$ (height $) \times 160$ $\mathrm{mm}$ (depth) and a weight of $8.9 \mathrm{~kg}$ was used (Figure 1). The device was equipped with an integrated water cleansing (using cold water) and two UV-C emitters (wavelength of $254 \mathrm{~nm}$; power requirement of $2 \times 13$ W). The UV-C emitters (equipped with splinter shields) and the electronics were integrated in the housing and waterproof. The housing consisted of stainless steel and of plastic at the top forming the three blade insertion positions 1 to 3 . After insertion, blades were automatically cleaned by the integrated cold-water jet and decontaminated by UV-C. The exposure time recommended by the manufacturer was $30 \mathrm{~s}$.

\section{Laboratory study with inoculated knives}

For knife inoculation, E. coli ATCC 
25922 and $S$. aureus ATCC 29213 strains were used. E. coli and S. aureus were selected as representatives of gram-negative and gram-positive bacteria, respectively. After growing of strains on plate count agar (Oxoid AG, Pratteln, Switzerland; $24 \mathrm{~h}$ at $37^{\circ} \mathrm{C}$ ), several E. coli or $S$. aureus colonies were collected with a sterile cotton swab and used for knife inoculation. Examined knives (stainless-steel blade) comprised two types of knives widely used in the meat industry (Swibo No. 5.8411.25 and 5.8411.26, Victorinox, Ibach-Schwyz, Switzerland). To evaluate the effect of UVC on inoculated $E$. coli or $S$. aureus, three experimental layouts were investigated: clean knives, knives contaminated with blood, and knives contaminated with fat. Clean knives were inoculated by rubbing the E. coli or $S$. aureus containing cotton swab in designated blade areas $\left(2 \times 5 \mathrm{~cm}^{2}\right.$ on each knife). After drying (about $5 \mathrm{~min}$ ), knives were inserted in the ME3-S device (Sterilsystems) and treated for $30 \mathrm{~s}$ (using positions 1 to 3 of the device) or for $45 \mathrm{~s}$ (using position 1 of the device). For the contamination experiments, sheep blood (Oxoid AG) and pork fat bought at retail were used. After inserting in sheep blood or rubbing over pork fat, cotton swabs were rubbed over designated blade areas $(2 \times 5$ $\mathrm{cm}^{2}$ on each knife). Blade areas were then inoculated with $E$. coli or $S$. aureus and further treated (UV-C for $30 \mathrm{~s}$ ) as described above.

Field study with naturally contaminated knives from a slaughterhouse

The UV-C ME3-S device (Sterilsystems) was used for knife decontamination in a Swiss slaughterhouse (slaughter capacity: $>20$ million $\mathrm{kg}$ per year). The UV-C device was thereby tested during the slaughter of cattle and sheep, both in the wet area (after removal of hooves) and in the clean area (after trimming) of the slaughterhouse. The examined, naturally contaminated knives (stainless-steel blades) were of various sizes and types. Knives were inserted in the UV-C device and treated for $30 \mathrm{~s}$ (using positions 1 to 3 of the device).

\section{Collection of samples}

Sampling was performed by swabbing: Sterile cotton swabs moistened with $0.85 \%$ saline solution were rubbed over designated blade areas before UV-C treatment (about 5 $\mathrm{cm}^{2}$ ) and after UV-C treatment (about 5 $\mathrm{cm}^{2}$ ). The number of replicates (before and after UV-C treatment) tested in the laboratory study for each $E$. coli and $S$. aureus was 100 for clean knives (50 for $30 \mathrm{~s}$ of exposure; 50 for $45 \mathrm{~s}$ of exposure), 16 for knives contaminated with blood, and 15 for knives contaminated with fat. In the field study examining naturally contaminated knives, 50 replicates from the wet area and 31 replicates from the clean area of the slaughterhouse were analyzed. Microbiological examinations were carried out immediately (laboratory study) or within $16 \mathrm{~h}$ (field study) after sampling.

\section{Microbiological examinations}

Samples were homogenized for $30 \mathrm{~s}$ in $5 \mathrm{~mL}$ of $0.85 \%$ saline solution in a stomacher, of which $1 \mathrm{~mL}$ was used to prepare decimal dilution series $(0.85 \%$ saline solution $)$. Aliquots of $0.1 \mathrm{~mL}$ were then transferred by the spreading method to plate count agars (Oxoid). Agars were used to determine the counts of E. coli and S. aureus $(24 \mathrm{~h}$ at $37^{\circ} \mathrm{C}$; laboratory study) or total viable counts (TVC; $72 \mathrm{~h}$ at $30^{\circ} \mathrm{C}$; field study). Counts were calculated as CFU cm$~^{-2}$ and the

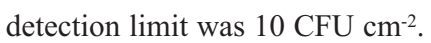

\section{Data analysis}

Colony counts were expressed as log $\mathrm{CFU} \mathrm{cm} \mathrm{cm}^{-2}$. A $\log$ value of zero was used for counts below the detection limit. For naturally contaminated knives, results were only considered when TVC before UV-C treatment were equal to or above the detection limit. Mean values and standard deviations before and after UV-C treatment and mean reductions obtained by UV-C treatment were then calculated. Statistical analysis was performed using JMP 13.0 (SAS Institute, Cary, NC, USA). The level of significance was set at $\alpha=0.05(\mathrm{P}<0.05)$. With regard to UV-C decontamination of knives, t-tests were used to analyze differences (i) between reductions of $E$. coli and $S$. aureus (30 s or $45 \mathrm{~s}$ of exposure; blood or fat con- tamination), (ii) between reductions after 30 $\mathrm{s}$ and $45 \mathrm{~s}$ of exposure (E. coli or S. aureus), (iii) between reductions on knives with and without additional contamination (blood or fat contamination, E. coli or S. aureus) or between reductions in the presence of blood and fat contamination (E. coli or $S$. aureus), and (iv) on naturally contaminated knives between TVC before and after UV-C treatment (wet or clean area) or between TVC from the wet and clean area (before and after UV-C and reductions). Moreover, analysis of variance (ANOVA) and the Tukey HSD test were used to analyze differences between reductions at the three positions of the UV-C device (knives with or without blood or fat contamination, E. coli or $S$. aureus; naturally contaminated knives from the clean or wet area).

\section{Results}

Reductions of inoculated E. coli and S. aureus on knives by UV-C treatment under laboratory conditions

In the laboratory experiments with inoculated knives (but lacking additional contaminations; Table 1), mean log counts before UV-C treatment were $5.98 \log$ CFU $\mathrm{cm}^{-2}$ for E. coli $(\mathrm{n}=50)$ and $6.65 \log \mathrm{CFU}$ $\mathrm{cm}^{-2}$ for $S$. aureus $(\mathrm{n}=50)$. Mean reductions obtained by UV-C treatment for $30 \mathrm{~s}$

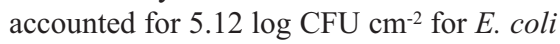

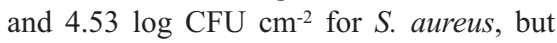
differences were not significant. With regard to the three insertion positions of the UV-C device (Figure 1), the lower reductions obtained at position 3 were striking $(\mathrm{P}<0.05)$, in particular as respective counts

Table 1. Decontamination of knives used in the meat industry with UV-C (ME3-S, Sterilsystems GmbH): Reductions of inoculated $E$. coli and $S$. aureus after 30s of UV-C exposure ( $n=50$ for each bacterial species).

\begin{tabular}{|c|c|c|c|c|c|}
\hline & & & & S. & eus $s^{a}$ \\
\hline & & $\bar{X}$ & SD & $\bar{X}$ & SD \\
\hline Before UV-C treatment & Total & 5.98 & 0.68 & 6.65 & 0.52 \\
\hline & Position $1^{\mathrm{b}}$ & 5.88 & 0.82 & 6.55 & 0.46 \\
\hline & Position 2 & 6.16 & 0.57 & 6.71 & 0.58 \\
\hline & Position 3 & 6.01 & 0.44 & 6.70 & 0.55 \\
\hline After UV-C treatment & Total $^{\mathrm{b}}$ & 0.86 & 1.53 & 2.12 & 2.19 \\
\hline & Position $1^{c}$ & 0.24 & 0.85 & 0.47 & 0.85 \\
\hline & Position 2 & 0.36 & 1.28 & 1.40 & 1.98 \\
\hline & Position 3 & 2.58 & 1.56 & 4.48 & 1.01 \\
\hline Mean log reductions by UV-C & Total & 5.12 & - & 4.53 & - \\
\hline & Position 1 & 5.64 & - & 6.08 & - \\
\hline & Position 2 & 5.80 & - & 5.31 & - \\
\hline & Position 3 & 3.43 & - & 2.22 & - \\
\hline
\end{tabular}

a $\overline{\mathrm{X}}$ and SD, mean $\log \mathrm{CFU} \mathrm{cm}{ }^{-2}$ and standard deviation; E. coli: position $1, \mathrm{n}=25$; position $2, \mathrm{n}=13$; position $3, \mathrm{n}=12$. S. aureus: position $1, \mathrm{n}=18$;

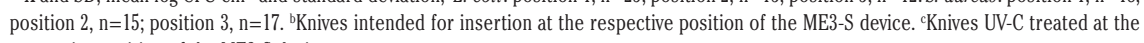
respective position of the ME3-S device. 
before UV-C treatment were comparable (Table 1). This could be due to the fact that the light intensity or the angel is different in this position. By increasing the UV-C exposure time to $45 \mathrm{~s}$, mean reductions account-

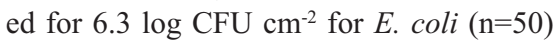
and $6.1 \log$ CFU cm$~^{-2}$ for $S$. aureus $(\mathrm{n}=50)$. Compared to the reductions after $30 \mathrm{~s}$ of UV-C exposure (and only considering results from position 1 of the UV-C device), $E$. coli reductions were increased on average by $0.7 \log \mathrm{CFU} \mathrm{cm} \mathrm{cm}^{-2}(\mathrm{P}<0.05)$, whereas $S$. aureus reductions were comparable.

The antibacterial effect of UV-C on inoculated knives with additional blood contamination ( $\mathrm{n}=16$ for each bacterial species) or fat contamination ( $\mathrm{n}=15$ for each bacterial species) was analyzed (Table 2). Compared to the results from knives without additional contamination, obtained reductions were clearly lower $(\mathrm{P}<0.05)$ and differed on average by 1.72 to $3.63 \log \mathrm{CFU}$ $\mathrm{cm}^{-2}$ from those of clean knives (depending on the type of contamination and the tested bacterial species). Fat contamination thereby showed a greater negative impact on the efficacy of UV-C than blood contamination $(\mathrm{P}<0.05$ for $E$. coli or $S$. aureus $)$. Reductions of $S$. aureus were lower $(\mathrm{P}<0.05)$ than those for $E$. coli in the presence of blood (on average by $0.9 \log \mathrm{CFU}$ $\mathrm{cm}^{-2}$ ), whereas reductions of $E$. coli and $S$. aureus were comparable in the presence of fat. With regard to the three insertion positions of the UV-C device (Figure 1), reduc-

Table 2. Decontamination of knives used in the meat industry with UV-C (ME3-S Sterilsystems $\mathrm{GmbH}$ ): Reductions of inoculated $E$. coli and $S$. aureus obtained on knives contaminated with blood (reflecting protein contamination) or fat after 30 s of UV-C exposure (using positions 1 to 3 of the device).

\begin{tabular}{|c|c|c|c|c|}
\hline & & & S. a & $1 S^{a}$ \\
\hline & $\bar{x}$ & SD & $\overline{\mathrm{X}}$ & SD \\
\hline Blood Contamination $(\mathrm{n}=16)$ & & & & \\
\hline Before UV-C treatment & 6.78 & 0.30 & 7.48 & 0.36 \\
\hline After UV-C treatment & 3.38 & 1.52 & 4.99 & 1.15 \\
\hline Mean log reductions by UV-C & 3.40 & - & 2.49 & - \\
\hline Fat Contamination $(\mathrm{n}=15)$ & & & & \\
\hline Before UV-C treatment & 6.86 & 0.50 & 7.34 & 0.26 \\
\hline After UV-C treatment & 5.37 & 1.67 & 5.62 & 0.95 \\
\hline Mean log reductions by UV-C & 1.49 & - & 1.72 & - \\
\hline
\end{tabular}

a $\overline{\mathrm{X}}$ and $\mathrm{SD}$, mean $\log \mathrm{CFU} \mathrm{cm}^{-2}$ and standard deviation.

Table 3. Decontamination of knives used in the meat industry with UV-C (ME3-S, Sterilsystems GmbH): Reductions of total viable counts (TVC) on naturally contaminated knives originating from the wet area $(n=50)$ and clean area $(n=24)$ of a slaughterhouse after 30s of UV-C exposure.a

\begin{tabular}{|c|c|c|c|c|}
\hline & & & S. & \\
\hline & $\bar{X}$ & SD & $\bar{X}$ & SD \\
\hline Before UV-C treatment & 3.75 & 0.89 & 1.52 & 0.59 \\
\hline After UV-C treatment & 2.97 & 0.71 & 0.89 & 1.05 \\
\hline Mean log reductions by UV-C & 0.78 & - & 0.63 & - \\
\hline
\end{tabular}

aTVC results from untreated and corresponding treated knife (from the clean area) only considered when TVC before UV-C treatment $\geq$ detection limit $(1.0 \log \mathrm{CFU} \mathrm{cm}-2){ }^{\mathrm{b}} \overline{\mathrm{X}}$ and SD, mean $\log \mathrm{CFU} \mathrm{cm}^{-2}$ and standard deviation.
After UV-C treatment, all 50 knives from the wet area and $12(50 \%)$ knives from the clean area still showed TVC above the detection limit (wet area: 1.6 to $4.6 \log \mathrm{CFU}$

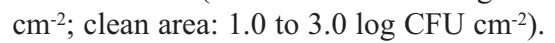

When comparing TVC reductions obtained by UV-C treatment on knives from the wet and clean area of a slaughterhouse, reductions were generally comparable and differed on average by less than $0.2 \log$ CFU $\mathrm{cm}^{-2}$ (despite the significant differences in the initial contamination, $\mathrm{P}<0.05$ ). With

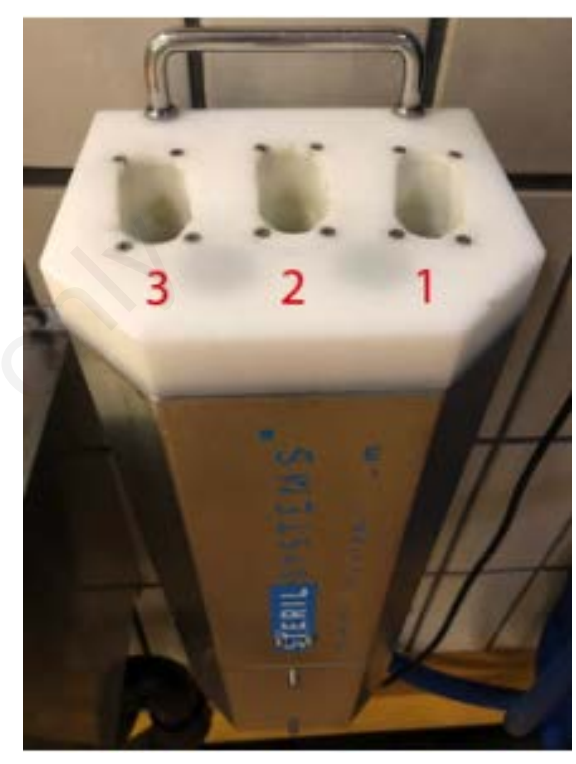

Figure 1. ME3-S device (Sterilsystems GmbH, Mauterndorf, Austria) for UV-C decontamination of knives with the different blade insertion sites (positions 1 to 3 ).

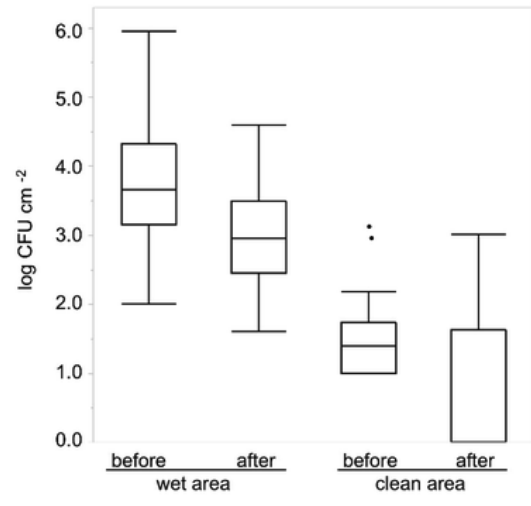

Figure 2. Total viable counts (TVC) on naturally contaminated knives from the wet area (after removal of hooves; $n=50$ ) and the clean area (after trimming; $n=24$ because results were only considered when TVC before UV-C treatment $\geq$ detection limit) of a slaughterhouse before and after UV-C treatment for 30s with the ME3-S device (Sterilsystems GmbH). 
regard to the three insertion positions of the UV-C device (Figure 1), TVC reductions tended to be comparable for knives from the wet or the clean area of the slaughterhouse, but comparisons were hampered by the limited and varying numbers of samples.

\section{Discussion and Conclusions}

Referred to the company, the ME3-S device was tested in accordance with the standard EN13679 and a disinfection effect of $>4 \log$ reduction on knives was achieved. In our study, under laboratory condition, without fat or protein, the obtained mean TVC reduction at a 30 s exposure time resulted in $5.1 \log$ CFU cm$~_{-2}$ for E. coli and $4.5 \mathrm{log}$

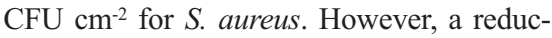
tion of the contamination of $>4 \log$ could not be achieved under running conditions in a slaughterhouse. The organic material can limit the effect of UV light in at least two ways: the bacteria get a place to hide, and aerosols and water around the knife after cleansing may form shadow zones for the light, and thereby lower the intensity of light exposure. Moreover, the time until enumeration was different for laboratory and industry knifes. There may be growth of bacteria between the sampling and enumeration for the abattoir knifes which could lead to a possible bias in these results. For the knife decontamination in slaughterhouses in Switzerland as well as in Europe a water disinfection method at a minimum of $82^{\circ} \mathrm{C}$ or an equivalent procedure is required (EU Regulation 853/2004). Eustace et al. (2008) recorded, that the TVC contamination on knives in slaughterhouses is reduced by an average of 1 to $2.5 \log$ steps using the current rinsing and sanitizing procedures. Bell and Hathaway (1996) found a mean TVC reduction of $2.6 \log \mathrm{CFU} \mathrm{\textrm {cm } ^ { - 2 }}$ by rinsing knives in warm water $\left(44^{\circ} \mathrm{C}\right)$ and immersing them in an $82^{\circ} \mathrm{C}$ water bath. Compared to other researches, our study showed a comparably mean TVC after the decontamination process with UV-C.

Leps et al. (2013) investigated, among others, a combination of water bath with ultrasound (in a laboratory setting). The use of ultrasound at $40^{\circ} \mathrm{C}$ and $50^{\circ} \mathrm{C}$ tempered water for $30 \mathrm{~s}$ affected the bacterial load only minimally (mean TVC reduction by ca. $\left.0.5 \log / 10 \mathrm{~cm}^{-2}\right)$, whereas an ultrasound treatment with $60^{\circ} \mathrm{C}$ for $30 \mathrm{~s}$ resulted in a mean TVC reduction of at least $4 \log \mathrm{CFU}$ $10 \mathrm{~cm}^{-2}$. Additionally, a combination of $40^{\circ} \mathrm{C}$ tempered water with lactic acid $(2 \%)$ showed an efficient way to eliminate microorganisms (mean TVC reduction of $2.3 \log \mathrm{CFU} 10 \mathrm{~cm}^{-2}$ in $5 \mathrm{~s}$ ). Brasil et al. (2017) evaluated the effect of ultrasound on knives in chlorinated water $\left(20^{\circ} / 25^{\circ} \mathrm{C}\right)$ with/without combination using neutral detergents. These authors concluded that a UV-C treatment seems not to be practicable if compared with the common method (hot water treatment at $82^{\circ} \mathrm{C}$ ).

Nevertheless, UV-C treatment has many advantages compared to the current procedure (low electricity cost and water consumption, no water steam). A future use of UV-C treatment might still be an option. Future studies should be performed to evaluate whether an effective prewash and a higher spray temperature in combination with UV-C treatment leads to more desirable outcomes in a commercial slaughterhouse setting.

\section{References}

Barbosa J, Cuppini M, Flach J, Steffens C, Cansian RL, Toniazzo G, 2016. Removal of Escherichia coli in boning knives with different sanitizers. Food Sci Technol 71;309-15.

Bell RG, Hathaway SC, 1996. The hygienic efficiency of conventional and inverted lamb dressing systems. J Appl Bacteriol 81:225-34.
Bintsis T, Litopoulou-Tzanetaki E, Robinson RK, 2000. Existing and potential applications of ultraviolet light in the food industry. J Sci Food Agric 80:637-45.

Brasil CC, Barin JS, Jacob-Lopes E, Menezes CR, Zepka LQ, Wagner R, Campagnol PC, Cichoski AJ, 2017. Single step non-thermal cleaning/sanitation of knives used in meat industry with ultrasound. Food Res Int 91:133-9.

Goulter RM, Dykes G, Small A, 2008. Decontamination of knives used in the meat industry: Effect of different water temperature and treatment on the reduction of bacterial numbers on knife surfaces. J Food Protect 71:1338-42.

EFSA/ECDC, European Food Safety Authority/European Center for Disease Prevention and Control, 2017. The European Union summary report on trends and sources of zoonoses, zoonotic agents and food-borne outbreaks in 2016. EFSA Journal, 50, 5077.

EU Regulation 853/2004. Regulation (EC) No. 853/2004 of the European Parliament and of the Council of 29 April 2004 laying down specific hygiene rules for food of animal origin.

Eustace I, Midgley J, Small A, Jenson I, Sumner J, 2008. Knife sanitizing in abattoirs: The effectiveness of current and alternative practices. Food Protect Trends 28:712-22.

Leps J, Einschütz K, Langkabel N, Fries R, 2013. Efficacy of knife disinfection techniques in meat processing. Meat Sci 95:185-9.

Nørrung B, Buncic S, 2008. Microbial safety of meat in the European Union. Meat Sci 78:14-24.

Sofos JN, 2008. Challenges to meat safety in the 21st century. Meat Sci 78:3-13.

Taormina J, Dorsa W, 2007. Evaluation of hot water and sanitizer dip treatments of knives contaminated with bacteria and meat residues. J Food Protect 70:648-54. 